\title{
REGISTER RERUM ad Vol. 39
}

Confecit G. Boehm, Basel

Adrenals, v. Nebennierenrinde

Anastomoses, v. Portosystemic communications

Anatomie comparée, v. Nagetiergehirn

Anatomie und Physiologie (Lehrbuch für ärztliches Hilfspersonal) (11. Aufl.)

(B) 187

Anthropologie des françaises, recherches (B)

Anthropology, v. Human body

Anthropometry, v. Human body

Antibody, fluorescent; localization of testicular hyaluronidase using

fluorescent antibody

189

Antigens, v. Antibody

Antikörper, v. Antibody

Aquatic rodents, v. Nagetiergehirn

Archaeopteryx, v. Squelette

Arcs branchiaux, v. Branchiometric nerves

Arcs viscéraux, v. Branchiomeric nerves

Autonomes Nervensystem (Vagus), v. Branchiomeric nerves

Aves; quelques caractères du squelette chez les oiseaux de la sous-classe des

Ratites 300

Beaver, v. Nagetiergehirn

Biber, v. Nagetiergehirn

Birds, v. Aves

Bisamratte, v. Nagetiergehirn

Body build, v. Human body

Bones, v. Squelette

Bovis, v. Hyaluronidase

Brain; connections of the temporal lobe in man

1

-; v. Cytoarchitectonique, Nagetiergehirn Branchial arches, v. Branchiomeric nerves Branchial bars; real or potential space between two branchial bars (=

«trema»), v. Branchiomeric nerves Branchial cleft, v. Branchiomeric nerves Branchies, v.

Branchiomeric nerves

Branchiomeric nerves, trematic interrelationships (R)

Bull testis, v. Hyaluronidase

Canis familiaris; portosystemic communications in the dog 271

Cardia, v. Portosystemic communications Carinates, v. Squelette

Carotid body tissue in the otter, distribution $\quad 259$

Castor, v. Nagetiergehirn

Castor canadensís, v. Nagetiergehirn 
Cavía porcellus, v. Elektroschock

«Cellules claires» (muqueuse uterine), v. «Helle Zellen»

$1(B)=$ Book reviews - Livres nouveaux - Buchbesprechungen $(\mathrm{R})=$ Survey - Revue générale Übersicht

352

Register rerum

Cerebralisation, v. Nagetiergehirn

Cerebrum; Beiträge zur vergleichenden Morphologie des Nagetiergehirnes, Supplementum 38 (= 1 ad Vol. 39)

-; connections of the temporal lobe in man

1

- ; etude des structures cytoarchitectoniques du diencéphale de Talpa

europaea (Insectivora, Talpidae)

90

Cerveau, v. Cerebrum

Chien, v. Canís famíliarís

Chicken, v. Hühnermagen

Chicken embryo, v. Hühnermagen

Cholesterin-Substanz der Nebennierenrinde bei experimentell ausgelöstem

epileptischem Anfall (Nebennierenrinde und Elektroschock II.) . . . 198

Cleft, gill cleft, v. Trematic interrelationships

Cobaye, v. Elektroschock

Cock, v. Sperm

Colon; lymphatic connections between the ascending and descending colon and the corresponding kidneys (Anatomical study) 264

-; v. Portosystemic communications

Comparative anatomy, v. Nagetiergehirn

Connections of the temporal lobe in man

Constitutional types, v. Human body

Coons method, v. Hyaluronidase

Coq v. Sperm

Corrigendum (ad Vol. 37 [1959] 163-192) 350

Cortex glandulae suprarenalis, v. Elektroschock

Coypou, v. Nagetiergehirn

Crane, nerves, v. Branchiomeric nerves

Cranial nerves, v. Branchiomeric nerves

Cytoarchitectonique; etude des structures cytoarchitectoniques du dien

céphale de Talpa europaea (Insectívora, Talpidae)

90

Cytochemistry, v. Hühnermagen, Hyaluronidase, Nebennierenrinde

Degeneration (nuclear degeneration, tract degeneration), v. Temporal lobe

Development, v. Ontogenesis

Diaphragma, v. Portosystemic communications

Diencephalon; etude des structures cytoarchitectoniques du diencéphale de

Talpa europaea (Insectivora, Talpidae)

90

Dinosaurian reptiles, v. Squelette

Dog; portosystemic communications in the dog

271

Drüsenepithel, v. Embryonaler Hühnermagen Duodenum, v. Portosystemic communications

Eiweiß-Schluckprozeß, v. Embryonaler Hühnermagen 
Elektroschock und Nebennierenrinde (II. Das Verhalten der CholesterinSubstanz der Nebennierenrinde bei experimentell ausgelöstem epi leptischem Anfall) 198 , (III. Das Verhalten der sudanophilen Substanzen der Nebennieren rinde beim experimentell ausgelösten epileptischen Anfall) 239 Register rerum 353

Embryonaler Hühnermagen, Epithel, histogenetische und histochemische Untersuchungen 51 Endometrium, v. «Helle Zellen» Entwicklung, v. Ontogenesis Epilepsy (lobectomy), v. Temporal lobe

Epileptic attacks, experimentally induced, v. Elektroschock

Epileptischer Anfall, v. Elektroschock

Epithel des embryonalen Hühnermagens, histogenetische und histochemi sche Untersuchungen 51

Epithel; über die «hellen Zellen» im Epithel der menschlichen Uterusschleimhaut 244

Estomac (embryon de poulet), v. Hühnermagen

Exstirpation des vorderen Schläfenlappens, v. Temporal lobe

Tacialis, v. Branchiomeric nerves

Fat, subcutaneous, v. Human body

Femmes, v. Françaises

Ferments, v. Embryonaler Hühnermagen, Hyaluronidase

Fettgewebe, subkutanes, v. Human body

Fischotter, v. Otter

Fluorescent antibody: localization of testicular hyaluronidase using fluorescent antibody 189

Foetus, v. Hühnermagen

Foie des mammifères hibernants. Anatomie et histologie 209

Foie, v. Portosystemic communications Fowl, v. Sperm

Françaises; recherches sur $\Gamma$ anthropologie des françaises (B)

Frauen, v. Françaises

Frog, v. Neuromuscular spindles

Frosch, v. Neuromuscular spindles

Fuseaux neuromusculaires, v. Neuromuscular spindles

Gallus gallus; histogenetische und histochemische Untersuchungen am

Epithel des embryonalen Hühnermagens

51

--; sperm morphology and viability

Gefäße, v. Carotid body, Portosystemic communications

Gefäße (Lymphgefäße), v. Lymphatic connections, Milz

Gehirn; Beiträge zur vergleichenden Morphologie des Nagetiergehirnes, Supplementum 38 (=1 ad Vol. 39)

-; v. Cytoarchitectonique, Temporal lobe

Geschlechtsmorphologie, v. Human body

Gill cleft, v. Branchiomeric nerves 
Glandula suprarenalis, v. Nebennierenrinde

Glandulär-zystische Hyperplasie (Uterusschleimhaut), v. «Helle Zellen»

Glandular stomach, epithelium, v. Embryonaler Hühnermagen

Glomus caroticum, v. Carotid body

Glossopharyngeus, v. Branchiomeric nerves

Glykogen, v. Embryonaler Hühnermagen

354

Register rerum

Grenouille, v. Neuromuscular spindles Guinea-pig, v. Elektroschock

Hahn, v. Sperm Handbuch, v. Primatologia

«Helle Zellen» im Epithel der menschlichen Uterusschleimhaut

Hepar, v. Foie, Portosystemic communications

Hibernating mammals, v. Hibernants

Hibernants, mammifères hibernants; le foie des mammifères hibernants.

Anatomie et histologie

Histochemische und histogenetische Untersuchungen am Epithel des em-

bryonalen Hühnermagens

51

Histochemistry, v. Hyaluronidase, Nebennierenrinde

Histogenetische und histochemische Untersuchungen am Epithel des em-

bryonalen Hühnermagens

51

Homínoidea, v. Primatologia Hühnchen, v. Hühnermagen Hühnermagen; histogenetische und histochemische Untersuchungen am

Epithel des embryonalen Hühnermagens

51

Huhn, v. Hühnermagen, Sperm

Human body; towards a systematic morphology of the human body . . . 220

Human brain, v. Temporal lobe

Hund, v. Canis familiarís

Hyaluronidase; localization of testicular hyaluronidase using fluorescent

antibody $\quad 189$

Hyperplasie glandulo-kystique (muqueuse uterine), v. «Helle Zellen» Hystricomorpha, v.

Nagetiergehirn

Infarct; right posterior temporo-parieto-occipital infarct (and infarction of

the inferior posterior right frontal region), v. Temporal lobe Insectivora, v. Talpa europaea

Intestinum crassum, v. Colon

Keratinoides Sekret, v. Embryonaler Hühnermagen

Kidney; lymphatic connections between the ascending and descending colon

and the corresponding kidneys (Anatomical study) 264

Kiemenbögen, v. Branchiomeric nerves Kiembogennerven, v. Branchiomeric nerves Knochen, v.

Squelette Körperbau, v. Human body Konstitutions-Typen, v. Human body

Leber, v. Foie, Portosystemic communications

Lehrbuch, v. Anatomie

Lesion (brain), v. Temporal lobe

Lien, v. Milz

Lipoide, v. Sudanophile Substanzen

Liver, v. Foie, Portosystemic communications

Lobectomy; right anterior temporal lobectomy, v. Lobus temporalis 
Register rerum 355

Lobus temporalis; connections of the temporal lobe in man

Loutre, v. Lutra lutra

Lutra lutra; distribution of carotid body tissue in the otter

Lymphatic connections between the ascending and descending colon and the corresponding kidneys (Anatomical study) Lymphgefäß-System der Milz 264

Magen, v. Embryonaler Hühnermagen Mammalia, v. Mammifères

Mammifères hibernants; leur foie. Anatomie et histologie 209

Man; connections of the temporal lobe in man 1

-; towards a systematic morphology of the human body 220

-; v. Branchiomeric nerves, Primatologia Manuel, v. Primatologia Maulwurf, v. Talpa europaea Meerschweinchen, v. Elektroschock Methode, v. Human body, Hyaluronidase

Milz, Lymphgefäß-System 84

Mitose (Prophase), v. «Helle Zellen» Mole, v. Talpa europaea

Morphology, systematic, of the human body 220

Mucosa uteri; über die «hellen Zellen» im Epithel der menschlichen Uterus-

schleimhaut 244

Muqueuse uterine, v. Mucosa uteri

Muscle, v. Neuromuscular spindles

Muscular stomach, epithelium, v. Embryonaler Hühnermagen

Musk-rat, v. Nagetiergehirn

Muskel, v. Muscle

Muskelspindeln, v. Neuromuscular spindles

Myocastor coypus, v. Nagetiergehirn

Myomorpha, v. Nagetiergehirn

Nagetiergehirn; Beiträge zur vergleichenden Morphologie des Nagetier-gehirnes, Supplementum 38 (= 1 ad Vol. 39)

Nebennierenrinde und Elektroschock (II. Das Verhalten der Cholesterin-

Substanz der Nebennierenrinde bei experimentell ausgelöstem epilep-

tischem Anfall) 198

; (III. Das Verhalten der sudanophilen Substanzen der Nebennieren

rinde beim experimentell ausgelösten epileptischen Anfall) 329

Nerven (Nervensystem, Zentralnervensystem), v. Diencephalon, Nagetiergehirn, Nerves, Neuromuscular spindles, Temporal lobe

Nerves; trematic interrelationships of the branchiomeric nerves (R) . . . .

Nervus facialis, v. Branchiomeric nerves

Nervus glossopharyngeus, v. Branchiomeric nerves

Nervus trigeminus, v. Branchiomeric nerves

Nervus vagus, v. Branchiomeric nerves

Neuromuscular spindles, living, morphological observations

Niere, v. Kidney

356 Register rerum

Nuclear degeneration, v. Temporal lobe Nutria, v. Nagetiergehirn

Oesophagus, v. Portosystemic communications

Oiseaux; quelques caractères du squelette chez les oiseaux de la sous-classe 
des Ratites

300

Omentum maius, v. Portosystemic communications Ondatra zibethica, v. Nagetiergehirn

Ontogenesis, v. Branchiomeric nerves Os, v. Squelette Osteologia, v. Squelette

Otter; distribution of carotid body tissue in the otter $\quad 259$

Ovalbumine, deglutition, v. Embryonaler Hühnermagen

Paraganglion, v. Carotid body

Parasympathicus, v. Branchiomeric nerves

Pfortader, v. Portosystemic communications

Pharyngeal arches, v. Branchiomeric nerves

Pharyngeal region, v. Branchiomeric nerves

Phosphatase, alkalische, v. Embryonaler Hühnermagen

187271270

Phylogenesis, v. Branchiomeric nerves

Physiologie und Anatomie (Lehrbuch für ärztliches Hilfspersonal) (11. Aufl.)

(B)

Polysaccharide, v. Embryonaler Hühnermagen Portal vein, v. Portosystemic communications

Portosystemic communications in the dog

Poulet, embryon, estomac, v. Embryonaler Hühnermagen Poultry, v. Sperm

Primatologia (Handbuch der Primatenkunde) (Vol. 1) (B)

Prophase (Mitose), v. «Helle Zellen» Proteins, v. Embryonaler Hühnermagen

Ramifications typiques du nerf branchial, v. Branchiomeric nerves

Rana, v. Neuromuscular spindles

Rat musqué, v. Nagetiergehirn

Rate, v. Milz

Ratites; quelques caractères du squelette chez les oiseaux de la sous-classe

des Ratites $\quad 300$

Rectum, v. Portosystemic communications

Ren, v. Kidney

Rein, v. Kidney

Reptiles dinosauriens, v. Squelette

Resection; anterior temporal resection, v. Temporal lobe

Rodentia; Beiträge zur vergleichenden Morphologie des Nagetiergehirnes,

Supplementum 38 (=1 ad Vol. 39) Rodents, v. Rodentia Rongeurs, v. Rodentia Rongeurs

aquatiques, v. Rodentia

Register rerum

357

Säugetiere, winterscblafende, v. Hibernants

Schädel, Nerven, v. Branchiomeric nerves

Schläfenlappen, vorderer, v. Temporal lobe

Schleimhaut (Uterus), v. «Helle Zellen»

Sciuromorpha, v. Nagetiergebirn

Semen, fowl semen, v. Sperm

Seminiferous epithelium, v. Hyaluronidase

Sexual morphology, v. Human body

Sinus caroticus, v. Carotid body

Skelet, v. Squelette 
Skeletal frame, v. Human body

Skull, nerves, v. Branchiomeric nerves

Soft tissues, development and distribution, v. Human body

Somatoskopie, v. Anthropologie, Human body

Sommeil hivernal, v. Hibernants

Sperm morphology and viability

Spermatocytes, v. Hyaluronidase

Spermatogonia, v. Hyaluronidase

Spermatozoa, v. Sperm

Spindles; morphological observations on living neuromuscular spindles . .

Spleen, v. Milz

Splen, v. Milz

Squelette; quelques caractères du squelette chez les oiseaux de la sous-classe

des Ratites $\quad 300$

Statistische Auswertung, v. Françaises, Human body

Stature (charpente squelettique), v. Human body

Stierhoden, v. Hyaluronidase

Stomach (chicken embryo), v. Hühnermagen

Sudanophile Substanzen der Nebennierenrinde, Verhalten beim experimentell ausgelösten epileptischen Anfall (Nebennierenrinde und Elektroschock

III.) $\quad 329$

Surrénales, v. Nebennierenrinde

Systematic morphology of the human body 220

Talpa europaea; etude des structures cytoarchitectoniques du diencéphale de

Talpa europaea (Insectivora, Talpidae) 90

Talpidae, v. Talpa europaea

Taupe, v. Talpa europaea

Taureau, testicule, v. Hyaluronidase

Temporal lobe in man, connections

Testicular hyaluronidase, localization, using fluorescent antibody ....

Thalamus, v. Temporal lobe

Tissu adipeux sous-cutané, v. Human body

Tissus mous, développement et distribution, v. Human body

Tract degeneration, v. Temporal lobe

«Trema» (= real or potential space between two branchial bars), v. Trematic interrelationship s 358

Register rerum

Trigeminus, v. Brancbiomeric nerves

Trematic interrelationships of the brancbiomeric nerves $(\mathrm{R})$

Uterus; über die «hellen Zellen» im Epithel der menschlichen Uterusschleim-

haut 244

Vagus, v. Brancbiomeric nerves

Vaisseaux, v. Carotid body, Portosystemic communications

Vaisseaux lymphatiques, v. Lymphatic connections, Milz

Vegetatives Nervensystem (Vagus), v. Branchiomeric nerves

Veins, v. Vena cava 
Vena azygos, v. Portosystemic communications

Vena cava; portosystemic communications in the dog

Vena portae; portosystemic communications in the dog

Venen, v. Portosystemic communications

Ventriculus, v. Embryonaler Hühnermagen, Portosystemic communications

Vergleichende Morphologie des Nagetiergehirnes, Beiträge, Supplementum 38

(= 1 ad Vol. 39) Vessels, v. Carotid body, Portosystemic communications Vessels (lymphatic), v.

Lymphatic connections, Milz Visceral arches, v. Branchiomeric nerves Vögel, v. Aves

Wassernager, v. Nagetiergehirn

Weichteile, Ausbildung und Verteilung, v. Human body

Winterschlaf, v. Hibernants

Women, v. Françaises

Zentralnervensystem der Nagetiere, vergleichende Anatomie, v. Nagetiergehirn Zwischenhirn, v.

Diencephalon Zyto..., v. Cyto...

Book Reviews - Lures Nouveaux - Buchbesprechungen

Bücker, J.: Anatomie und Physiologie (Lehrbuch für ärztliches Hilfsperso-

nal) (11. Aufl.) (Verlag G. Thieme, Stuttgart 1959)

187

Felice, Suzanne de: Recherches sur l'anthropologie des françaises (Masson \&

Cie., Paris 1958)

187

Hofer, H., A. H. Schultz und D. Starck (Editores): Primatologia (Handbuch

der Primatenkunde) Vol. I (Verlag S. Karger AG. Basel/New York 1956) 270

News from universities - Nouvelles universitaires - Universitätsnachrichten

Bucher, $0 \quad 188$

Popoff,N. $\quad 188$

Wolf-Heidegger, G $\quad 188$

Zawísch, Carla v $\quad 188$ 